\title{
Intestinal Co-culture System to Study TGR5 Agonism and Gut Restriction
}

\author{
Snehal N. Chaudhari* and A. Sloan Devlin*
}

Department of Biological Chemistry and Molecular Pharmacology, Harvard Medical School, Boston, USA

*For correspondence: snehal chaudhari@hms.harvard.edu; sloan devlin@hms.harvard.edu

[Abstract] The activation of the Takeda G-protein receptor 5 (TGR5, also known as the G proteincoupled bile acid receptor 1, GPBAR1) in enteroendocrine L-cells results in secretion of the anti-diabetic hormone Glucagon-Like Peptide 1 (GLP-1) into systemic circulation. Consequently, recent research has focused on identification and development of TGR5 agonists as type 2 diabetes therapeutics. However, the clinical application of TGR5 agonists has been hampered by side effects of these compounds that primarily result from their absorption into circulation. Here we describe an in vitro screening protocol to evaluate the TGR5 agonism, GLP-1 secretion, and gut-restricted properties of small molecules. The protocol involves differentiating gut epithelial and endocrine cells together in transwells to assess both the pharmacodynamics of TGR5 agonists and the toxicity of compounds to the intestinal monolayer. As a proof of concept, we demonstrate the use of the protocol in evaluating properties of naturally occurring bile acid metabolites that are potent TGR5 agonists. This protocol is adapted from Chaudhari et al. (2021).

Keywords: Enteroendocrine cells, TGR5, GLP-1, Gut-restriction, Intestinal permeability, Diabetes

[Background] The intestinal wall of the GI tract is comprised of several different types of cells, each with specific and, sometimes exclusive functions (Allaire et al., 2018). In vitro testing of small molecule therapeutics or drug candidates has traditionally relied on mono-enteric cell lines. These in vitro systems do not determine the absorptive properties of the small molecule being tested, nor do they predict the behavior of these compounds in the gut. To some extent, enteroids recapitulate the complexity and diversity of the intestine, but they are an expensive system and do not always provide reproducible results due to variability in the reagents required (Blutt et al., 2018). There is an unmet need for simple experimental systems that mimic the gut epithelial layer to aid in the development of new chemical probes and drugs. Here, we focus on developing cell-based protocols to assay one intestinal signaling system, the small molecule-based activation of the G protein-coupled receptor TGR5 on intestinal enteroendocrine cells.

The study of small molecule agonists of TGR5 has gained significant importance in recent years due to the potential therapeutic benefits of these compounds, primarily in diabetes control (Duboc et al., 2014). TGR5 is expressed on enteroendocrine L-cells in the lower gastrointestinal ( $\mathrm{Gl}$ ) tract, and when activated, results in secretion of the anti-diabetic hormone GLP-1 into the bloodstream (Eissele et al., 1992; Harach et al., 2012). Activation of this gut-initiated TGR5-GLP1 cascade shows promise as a potential target for type 2 diabetes therapeutics (Hodge and Nunez, 2016). However, activation of TGR5 
outside of the gut can cause unwanted side effects (Cao et al., 2016; Hodge and Nunez, 2016). Therefore, a unified in vitro screening method that can be used to evaluate the effects of small molecules on TGR5 activation and GLP-1 secretion, as well as the interaction of these compounds with the gut epithelial barrier, will aid in the development of successful new TGR5-targeted therapies.

Here we describe a simple in vitro protocol from Chaudhari et al. (2021) to co-differentiate human intestinal epithelial cells with human enteroendocrine cells into a polarized monolayer in transwells. We then describe how to utilize the resulting epithelial layer to test the TGR5 agonism and gut restrictedproperties of small molecules. Our system incorporates GLP-1-secreting NCl-H716 L-cells, which have been the predominant cell line used to test TGR5 activation and GLP-1 secretion (Kim et al., 2017; Sato et al., 2008), into an epithelial monolayer formed by surrounding human intestinal colon carcinoma Caco-2 epithelial cells. This monolayer forms a physical and biochemical barrier to small molecules.

Through electron microscopy imaging, we show that the enteroendocrine cells intercalate with the colon epithelial cells and are polarized, with distinct apical and basolateral faces. Mass spectrometrybased quantification of the passage of small molecules from the apical to the basolateral chamber through the differentiated monolayer allows for evaluation of the gut-restricted properties of the compounds (Chaudhari et al., 2021). Using this system, we can also perform RNA interference knockdowns, harvest cells to extract RNA and quantify TGR5 expression, and measure GLP-1 secretion. Importantly, because small molecules can be added to either face of the differentiated monolayer, information about whether compounds can induce TGR5 activation and expression and GLP-1 secretion from the apical face, basolateral face, or both faces, can be determined. Lastly, we describe how this system can be used to study toxic effects of small molecules on intestinal cells as well as the effects of these compounds on epithelial barrier integrity. This in vitro co-culture transwell system will enable the identification of small molecules that are both TGR5 agonists and possess limited off-targeted effects and will thus facilitate the development of novel type 2 diabetes therapies.

\section{Materials and Reagents}

1. TC treated flasks (Genesee, catalog number: 25-210)

2. Olympus vacuum filter systems, $500 \mathrm{ml}$ (Genesee, catalog number: 25-227)

3. Falcon $15 \mathrm{ml}$ conical centrifuge tubes (Fisher Scientific, catalog number: 14-959-53A)

4. Falcon $50 \mathrm{ml}$ conical centrifuge tubes (Fisher Scientific, catalog number: 14-432-22)

5. Corning Transwell polycarbonate membrane cell culture inserts (Millipore Sigma, catalog number: CLS3401-48EA)

6. RNase-free microfuge tubes (1.5 ml) (Fisher Scientific, catalog number: AM12400)

7. Precellys lysing tubes VK05, 2 ml (Bertin Instruments, catalog number: P000913-LYSKO-A)

8. Sep-Pak C-18 $40 \mathrm{mg}$ 96-well pate (Waters, catalog number: 186003966)

9. Fisherbrand 96-Well DeepWell polypropylene microplates (Fisher Scientific, catalog number: 12-566-120) 
10. Corning 96-well solid black polystyrene microplates (Fisher Scientific, catalog number: 07-200590)

11. Human colorectal adenocarcinoma cell line Caco-2 (ATCC, catalog number: HTB-37)

12. Human colorectal adenocarcinoma cell line NCl-H716 (ATCC, catalog number: CCL-251)

13. DMEM, high glucose, with L-glutamine (Genesee, catalog number: 25-500)

14. RPMI 1640, with L-glutamine and phenol red (Genesee, catalog number: 25-506)

15. GenClone fetal bovine serum (Genesee, catalog number: 25-514)

16. Penicillin-streptomycin 100x solution (Genesee, catalog number: $25-512$ )

17. Trypsin-EDTA, $0.25 \% 1 \times$, phenol red (Genesee, catalog number: $25-510$ )

18. Gibco Trypan blue solution, $0.4 \%$ (Thermo Fisher Scientific, catalog number: 15250061)

19. Fluorescein isothiocyanate-dextran 4 kDa (Millipore Sigma, catalog number: 46944-100MG-F)

20. Opti-MEM reduced serum media, GlutaMAX Supplement (Thermo Fisher Scientific, catalog number: 51985091)

21. Lipofectamine 3000 transfection reagent (Thermo Fisher Scientific, catalog number: L3000001)

22. PBS $1 \times$ (Genesee, catalog number: 25-507)

23. Trifluoroacetic acid (TFA) (Millipore Sigma, catalog number: T6508)

24. Hydrochloric acid ( $\mathrm{HCl})$ (Millipore Sigma, catalog number: H1758)

25. Formic acid (Millipore Sigma, catalog number: F0507)

26. $\mathrm{NaCl}$ (Millipore Sigma, catalog number: S9888)

27. Isopropyl alcohol (Millipore Sigma, catalog number: 563935)

28. GLP-1 EIA Kit (Millipore Sigma, catalog number: RAB0201-1KT)

29. TRIzol reagent (Thermo Fisher Scientific catalog number: 155626)

30. Promega CellTiter-Glo luminescent cell viability assay kit (Promega, catalog number: PR-G7570)

31. MTT Assay for cell viability and proliferation (Millipore Sigma, catalog number: 11465007001)

32. Complete media (see Recipes)

33. Transfection media (see Recipes)

34. Low-serum media for GLP-1 secretion (see Recipes)

35. Cell lysis buffer (see Recipes)

\section{Equipment}

1. Hemocytometer for cell counting (Weber Scientific, catalog number: 3048-12)

2. $-80^{\circ} \mathrm{C}$ freezer

3. Biosafety cabinet

4. Water bath $\left(37^{\circ} \mathrm{C}\right)$

5. Tissue culture incubator $\left(37^{\circ} \mathrm{C}, 5 \% \mathrm{CO}_{2}\right)$

6. Inverted microscope (Amscope)

7. Bench-top centrifuge

8. Fluorescence and absorbance plate reader (FlexStation 3, Molecular Devices) 
9. MagNA Lyser (Roche Diagnostics)

10. SpeedVac vacuum concentrators (Thermo Fisher Scientific)

11. Ultra-high performance liquid chromatography-mass spectrometry (UPLC-MS) with a 6120 Quadrupole LC/MS spectrometer (Agilent Technologies)

12. qPCR instrument (QuantStudio 7 Pro, Thermo Fisher Scientific)

\section{Software}

1. SoftMax Pro 7 (Molecular Devices, http://www.moleculardevices.com)

2. OpenLab Chromatography Data System (Agilent Technologies)

3. Applied Biosystems qPCR analysis software (QuantStudio 7 Pro, Thermo Fisher Scientific)

4. Microsoft Excel and GraphPad Prism 7 (https://www.graphpad.com/scientific-software/prism/) for data plotting and analyses

\section{Procedure}

A. Grow cells to confluency

1. Prepare and filter-sterilize complete DMEM and RPMI media (Recipe 1). Warm media to $37^{\circ} \mathrm{C}$ prior to use.

2. Thaw the attachment cell line Caco-2 and resuspend in complete DMEM media (Gagnon and Brubaker, 2015). Thaw the suspension cell line NCl-H716 and resuspend in complete RPMI media (Gagnon and Brubaker, 2015; Lea, 2015).

3. Seed both cell lines separately into TC treated flasks (seeding density for Caco-2 cells, $1 \times 10^{4}$ cells per $\mathrm{cm}^{2} ; \mathrm{NCl}-\mathrm{H} 716$ cells, $4 \times 10^{5}-8 \times 10^{5}$ cells per ml) and immediately transfer them to the tissue culture incubator $\left(5 \% \mathrm{CO}_{2}, 37^{\circ} \mathrm{C}\right)$. Media added at $0.2 \mathrm{ml} \mathrm{per} \mathrm{cm}^{2}$.

4. Passage Caco- 2 cells by first detaching cells with $1 \times \operatorname{Trypsin}$-EDTA $\left(1 \mathrm{ml}\right.$ per $\left.\mathrm{cm}^{2}\right)$ for no more than $10 \mathrm{~min}$ in the tissue culture incubator. Once cells are detached, add complete DMEM media to the flask to deactivate the Trypsin enzyme, followed by resuspension in complete DMEM media in the flask and transfer to flasks at a subcultivation ratio of 1:5, followed by incubation in the tissue culture incubator. Passage $\mathrm{NCl}-\mathrm{H} 716$ cells by collection of cells in Falcon tubes and centrifugation at $500 \times \mathrm{g}, 5 \mathrm{~min}$, room temperature. Remove supernatant and resuspend cells in fresh complete RPMI media, transfer to flasks at a subcultivation ratio of $1: 5$, followed by incubation in the tissue culture incubator.

5. Grow Caco-2 and NCl-H716 cells in TC Treated Flasks for up to 2 weeks to obtain desired number of cells for seeding and differentiation (see Step A3). Thaw the appropriate number of cells in Step A2 such that the required number of cells are obtained in 2 weeks, which is the optimal growth time for robust differentiation. Cells should not be passaged more than 15 times to avoid heterogeneity in chromosome distribution, which could affect differentiation and gene 
expression in the cell lines. Count Caco-2 and $\mathrm{NCl}-\mathrm{H} 716$ cells in a hemocytometer using Trypan blue staining. Once desired confluency is reached, proceed to differentiation step.

Note: For a 24-well transwell, 200,000 Caco-2 cells and 50,000 NCl-H716 cells will be required to initiate differentiation (see Step B3). Therefore, with 1 confluent $75 \mathrm{~cm}^{2}$ TC Treated Flask of each cell line, approximately 40 24-well transwells can be seeded for differentiation.

B. Seed and differentiate cells in Transwells

1. To start differentiation and co-culture, Trypsinize and collect Caco-2 cells, and collect $\mathrm{NCl}$ $\mathrm{H} 716$ cells in $50 \mathrm{ml}$ Falcon tubes, followed by centrifugation ( $500 \times \mathrm{g}, 5 \mathrm{~min}$, room temperature).

2. Remove supernatant and resuspend cells in desired volume of fresh complete DMEM media.

3. Mix 200,000 Caco-2 cells with $50,000 \mathrm{NCl}-\mathrm{H} 716$ cells in Falcon tubes, and seed in each 24-well plate transwell inserts to mimic the ratio of epithelial cells to enteroendocrine cells in the human colon (Cristina et al., 1978). The final volume of media and cells in each transwell insert should be $100 \mu \mathrm{l}$, and the final number of cells seeded is 250,000 per transwell insert.

4. Add $500 \mu \mathrm{l}$ of complete DMEM media in the basolateral chamber of the transwell inserts. Use DMEM media throughout differentiation. Transfer plates to the tissue culture incubator for differentiation (Day 1).

5. Replace media on Days 4, 8, 12, 16, and 18 to differentiate cells (Lea, 2015). To change media, carefully remove media, first from the apical compartment of the transwells (without disturbing or touching the seeded cells), then from the basolateral chamber. Replace with fresh complete pre-warmed DMEM media, first by adding $100 \mu$ media to the apical chamber, then by adding $500 \mu \mathrm{l}$ media to the basolateral chamber of the transwell inserts.

6. Differentiation of cells will take 21 days. On Day 21 , differentiated polarized cells are ready for assays (Figure 1), and must be utilized by Day 27. 


\section{bĭ̈-protocol

A
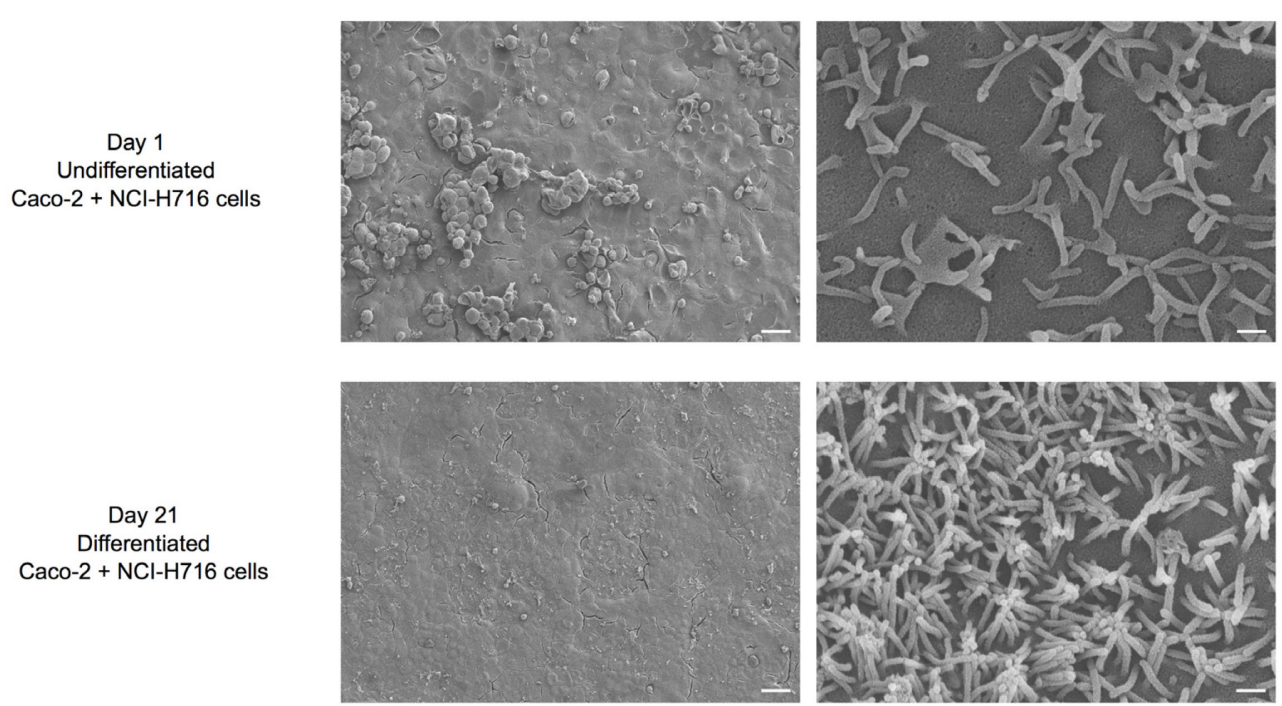

B

C

D
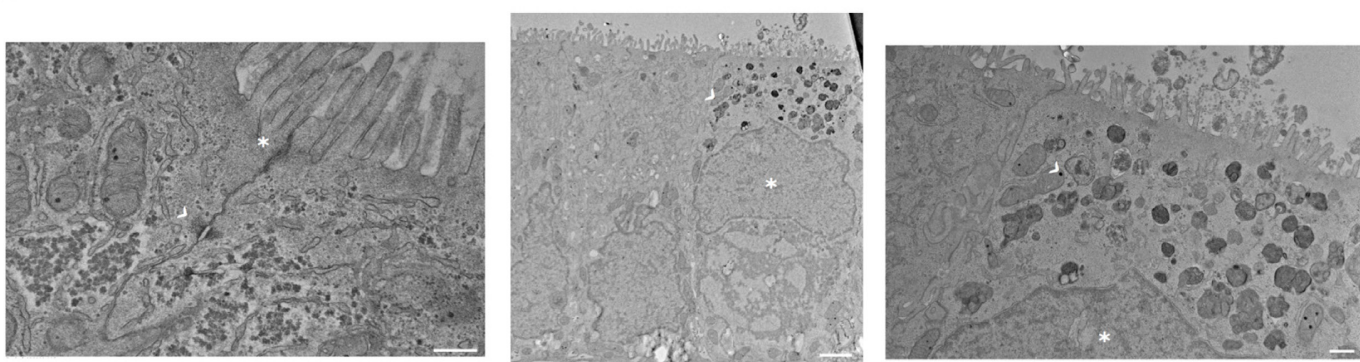

Figure 1. Electron microscopy images of intestinal cells in transwells. A. Scanning electron microscopy (SEM) images of Day 1 (undifferentiated) and Day 21 (differentiated) Caco$2+\mathrm{NCl}-\mathrm{H} 716$ cells. Differentiated cells have a monolayer of cells with distinct microvilli. Scale bars from left to right, $400 \mu \mathrm{m}$ and $4 \mu \mathrm{m}$. B. Transmission electron microscopy (TEM) images of differentiated Caco-2 $+\mathrm{NCl}-\mathrm{H} 716$ cells. Asterisk $\left({ }^{*}\right)$ shows tight junctions between adjacent cells, arrow (>) show desmosomes. Scale bar, $400 \mathrm{~nm}$. C. Transmission electron microscopy (TEM) images of differentiated Caco-2 + NCl-H716 cells. The cell on the left is a Caco-2 epithelium cell, while the cell on the right is a $\mathrm{NCl}-\mathrm{H} 716$ enteroendocrine cell with relatively displaced nucleus $\left(^{*}\right)$ and presence of dark granules (>) (Gonzalez et al., 2013). Scale bar, $400 \mathrm{~nm}$. D. Higher-magnification of the $\mathrm{NCl}-\mathrm{H} 716$ cell from (C) showing structures of the dark granules (>). Asterisk shows the nucleus $\left({ }^{*}\right)$. Scale bar, $2 \mu \mathrm{m}$. Electron microscopy performed by the EM core at Harvard Medical School.

C. Transfection of differentiated Caco-2 $+\mathrm{NCl}-\mathrm{H} 716$ cells with siRNAs or shRNA plasmids

1. Prepare desired concentration of siRNAs or shRNA plasmids (for example, $40 \mathrm{nM}$ ) in low serum media such as OptiMEM with a transfection reagent such as Lipofectamine 3000 (Recipe 2). Perform transfection according to manufacturer's instructions on Day 19 of differentiation.

2. Perform first siRNA or shRNA treatment by adding siRNA or shRNA plasmids in low-serum and antibiotic-free media to the apical chamber at $100 \mu \mathrm{l}$ and the basolateral chamber at $500 \mu \mathrm{l}$ and incubate for $12 \mathrm{~h}$. 
3. After the $12 \mathrm{~h}$ incubation, replace media with complete DMEM media for $12 \mathrm{~h}$.

4. After the $12 \mathrm{~h}$ incubation, on Day 20 of differentiation, perform second siRNA or shRNA treatment by adding siRNA or shRNA plasmid in low-serum and antibiotic-free media such as OptiMEM to the apical chamber at $100 \mu \mathrm{l}$ and the basolateral chamber at $500 \mu \mathrm{l}$ and allow to incubate for $12 \mathrm{~h}$.

5. After the $12 \mathrm{~h}$ incubation, replace media with complete DMEM media for $12 \mathrm{~h}$.

6. On Day 21, siRNA- or shRNA-treated differentiated polarized cells are ready for assays, and must be utilized by Day 23 .

D. Treatment of differentiated cells with small molecules such as bile acids for GLP-1 secretion assay

1. Prepare concentrated molecule stocks to be tested in the desired concentrations in a suitable vehicle such as DMSO or PBS. Use appropriate vehicle as negative control. Do not use $>10 \%$ DMSO solution to minimize toxic effects of DMSO on the cells and the monolayer integrity.

2. Prepare low serum RPMI media (0.5\% FBS) (Recipe 3 ) without antibiotics, and prepare dilutions of molecules in this media (Gagnon and Brubaker, 2015).

3. On Day 21 of the differentiation, remove media from both the apical and basolateral compartments of the transwells.

4. To test if molecules can induce GLP-1 secretion when treated apically, add molecules diluted in low serum RPMI media (Gagnon and Brubaker, 2015) to the apical chamber of the transwell inserts in a total volume of $100 \mu \mathrm{l}$. Add $500 \mu \mathrm{l}$ of low serum RPMI media to the basolateral chamber. The monolayer would prevent diffusion of media between the chambers.

5. To test if molecules can induce GLP-1 secretion when treated basolaterally, add molecules diluted in low serum RPMI media to the basolateral chamber of the transwell inserts in a total volume of $500 \mu \mathrm{l}$. Add $100 \mu \mathrm{l}$ of low serum RPMI media to the apical chamber.

6. Incubate in the tissue culture incubator for $2 \mathrm{~h}$.

7. During the incubation, prepare Microfuge tubes with 1\% TFA in water to collect basolateral media such that the final concentration of TFA will be $0.1 \%$. Keep on ice. For example, for $1 \mathrm{ml}$ of media to be collected, prepare tubes with $10 \mu$ of $1 \%$ TFA on ice.

8. Prepare cell lysis buffer (Recipe 4) fresh and keep on ice.

9. After the incubation period, collect basolateral media and transfer to prepared Microfuge tubes with TFA.

10. Remove apical media, add $200 \mu \mathrm{l}$ of cold cell lysis buffer (Recipe 4) to each transwell. Collect cells by gently scraping the transwells, and transfer contents into Precellys lysing tubes with beads.

11. Lyse cells by homogenization in a MagNA Lyser at $2,800 \times g$ for $1 \mathrm{~min}$, ideally in a cold room to prevent heating of the samples during homogenization.

12. Freeze collected cells and media at $-80^{\circ} \mathrm{C}$ for future analysis described in $\mathrm{E}$. Alternate protocols for GLP-1 peptide purification and measurement can also be used. 
E. GLP-1 peptide purification and quantification (for ELISA-based detection of GLP-1)

1. Thaw frozen samples on ice.

2. Centrifuge media at $1,800 \times g, 10 \mathrm{~min}$ at $4{ }^{\circ} \mathrm{C}$ to pellet cell debris.

3. During the centrifugation, prepare GLP-1 peptide purification and elution buffers. Buffer $A$ (wash buffer) is $0.1 \%$ TFA in water, buffer $B$ (elution buffer) is $0.1 \%$ TFA in $80 \%$ isopropyl alcohol in water.

4. Pretreat Sep-Pak columns by adding $500 \mu$ l of buffer B. Allow buffer to drip through the columns or use air pressure or centrifugation at $11,000 \times g, 1 \mathrm{~min}$ to force the buffer through the columns. Discard eluate.

5. Equilibrate Sep-Pak columns by adding $500 \mu \mathrm{l}$ of buffer A. Allow buffer to drip through the columns or use air pressure or centrifugation at $11,000 \times \mathrm{g}, 1 \mathrm{~min}$ to force the buffer through the columns. Discard eluate.

6. Load columns with media or cell lysate at 200-300 $\mu \mathrm{l}$. Incubate at room temperature for $5 \mathrm{~min}$.

7. Allow buffer to drip through the columns or use air pressure or centrifugation at $11,000 \times g, 1$ min to force the buffer through the column. Discard eluate.

8. Wash by adding $500 \mu \mathrm{l}$ of buffer A. Allow buffer to drip through the columns or use air pressure or centrifugation at $11,000 \times \mathrm{g}, 1 \mathrm{~min}$ to force the buffer through the columns. Discard eluate.

9. Elute GLP-1 peptide by adding $500 \mu \mathrm{l}$ of buffer B. Allow buffer to drip through the columns or use air pressure or centrifugation at $11,000 \times \mathrm{g}, 1 \mathrm{~min}$ to force the buffer through the columns. Collect the eluate in a 96-well plate directly by allowing flow-through to drip into wells.

10. Dry the eluate containing purified GLP-1 peptides in a SpeedVac for $4 \mathrm{~h}$.

11. Resuspend in $100 \mu \mathrm{l} 0.1 \%$ TFA in water and freeze at $-80^{\circ} \mathrm{C}$ for GLP-1 measurement analysis.

12. Use purified peptides in GLP-1 EIA Kit to measure total GLP-1 levels according to manufacturer's instructions with $0.1 \%$ TFA in water as a negative control in addition to vehicle control used for normalization (Figure 2A). The following calculation may be used to determine percentage GLP-1 secretion:

Percentage GLP-1 secretion $=($ Total GLP-1 secreted into media $) /($ Total GLP-1 secreted into media + Total GLP-1 in cell lysates) $\times 100$.

F. Treatment of differentiated cells with molecules for induction of TGR5 expression

1. Prepare concentrated molecule stocks to be tested in the desired concentrations in a suitable vehicle such as DMSO or PBS. Use appropriate vehicle as negative control. Do not use $>10 \%$ DMSO solution to minimize toxic effects of DMSO on the cells and the monolayer integrity.

2. Prepare dilutions of molecules in complete DMEM media.

3. On Day 21 of the differentiation, remove media from the apical and basolateral compartment of the transwells.

4. To test if molecules (here we test bile acid metabolites) can induce TGR5 expression when treated apically, add molecules diluted in complete DMEM media to the apical chamber of the 
transwell inserts in a total volume of $100 \mu \mathrm{l}$. Add $500 \mu \mathrm{l}$ complete DMEM media to the basolateral chamber.

5. To test if molecules can induce TGR5 expression when treated basolaterally, add molecules diluted in complete DMEM media to the basolateral chamber of the transwell inserts in a total volume of $500 \mu \mathrm{l}$. Add $100 \mu \mathrm{l}$ of complete DMEM media to the apical chamber. Complete DMEM media is used here to provide cells with optimal FBS and glucose so that the monolayer integrity is not compromised during the overnight incubation in the next step.

6. Incubate in the tissue culture incubator overnight (up to $16 \mathrm{~h}$ ).

7. After the incubation period, remove media from the apical and basolateral chambers and wash transwells gently with PBS.

8. Add TRIZol or other appropriate buffers directly to the transwells for RNA extraction, and scrape cells to collect into Microfuge tubes.

9. Freeze at $-80{ }^{\circ} \mathrm{C}$ for future RNA extraction and $\mathrm{qPCR}$ analysis (Figure $2 \mathrm{~B}$ ).

A
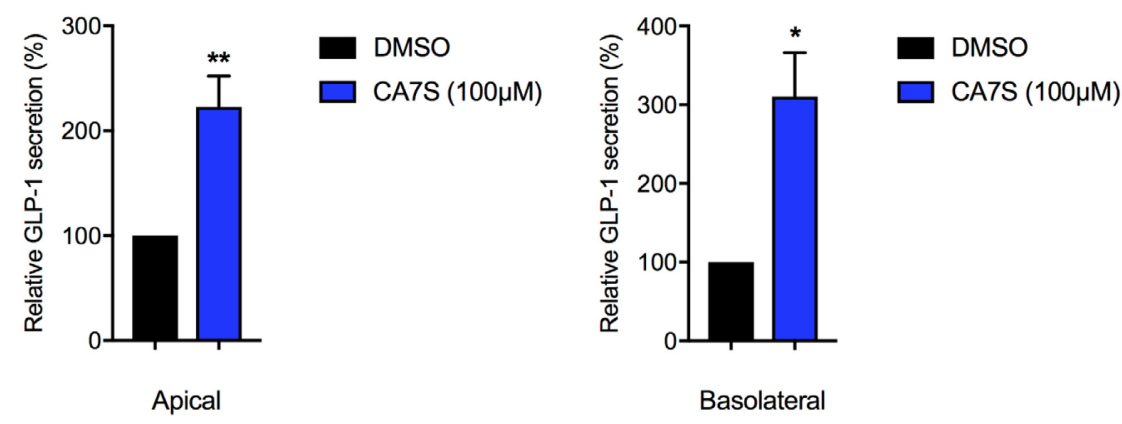

B
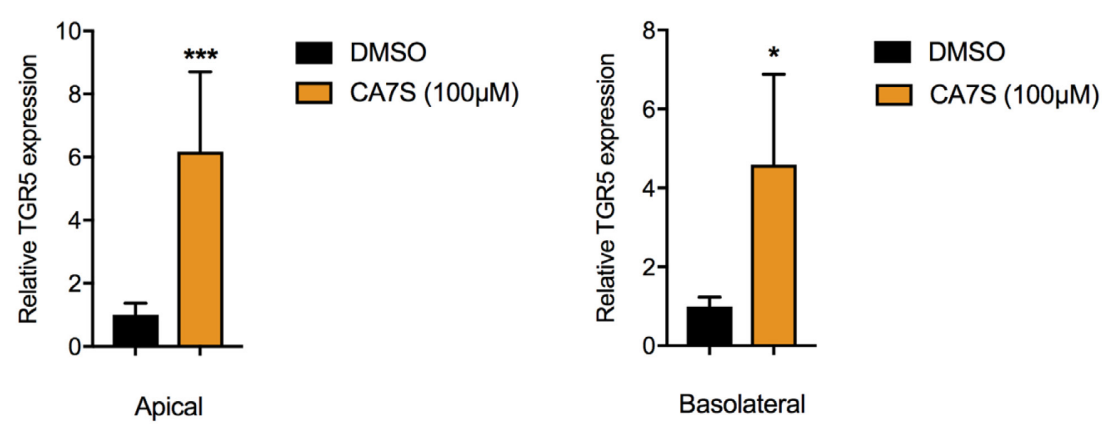

Figure 2. Testing of small molecule TGR5 agonists for induction of TGR5 expression and GLP-1 secretion using the co-culture transwell system. A-B. Cholic acid-7-sulfate (CA7S), a naturally occurring TGR5 agonist and GLP-1 secretagogue induces GLP-1 secretion (A) and TGR5 expression (B) in differentiated Caco-2 $+\mathrm{NCl}-\mathrm{H} 716$ cells when treated apically or basolaterally, similar to previous observations (Chaudhari et al., 2021). ( $\geq 3$ biological replicates per condition, CA7S Apical GLP-1, ${ }^{* *} P=8 \times 30^{-3}$; CA7S Basolateral GLP-1, ${ }^{*} P=0.03$; CA7S Apical TGR5, ${ }^{* *} P=3 \times 10^{-4}$; CA7S Basolateral TGR5, ${ }^{*} P=0.01$, Welch's $t$-test). No detectable induction of GLP-1 or TGR5 expression was observed in differentiated Caco-2 cells alone. GLP- 
1 absorbance readings and qPCR analysis were performed as described previously (Chaudhari et al., 2021) at the ICCB-Longwood Screening facility at Harvard Medical School. See Data analysis section for calculation of relative GLP-1 secretion in A. All data are presented as mean \pm SEM.

G. Treatment of differentiated cells with molecules for determination of gut-restricted properties, cell toxicity, and effect on intestinal permeability

1. Prepare concentrated molecule stocks to be tested in the desired concentrations in a suitable vehicle such as DMSO or PBS. Use appropriate vehicle as negative control. Do not use $>10 \%$ DMSO solution to minimize toxic effects of DMSO on the cells and the monolayer integrity.

2. Prepare dilutions of molecules in complete DMEM media.

3. On Day 21 of the differentiation, remove media from the apical and basolateral compartment of the transwells.

4. To test if molecules are gut-restricted, add molecules diluted in complete DMEM media to the apical chamber of the transwell inserts in a total volume of $100 \mu \mathrm{l}$. Add $500 \mu \mathrm{l}$ complete DMEM media to the basolateral chamber.

5. Incubate in the tissue culture incubator overnight (up to $16 \mathrm{~h}$ ).

6. After the incubation period, collect basolateral media in Microfuge tubes.

7. Detect and quantify molecules in basolateral media using appropriate instruments and assays (Figure 3). 
A

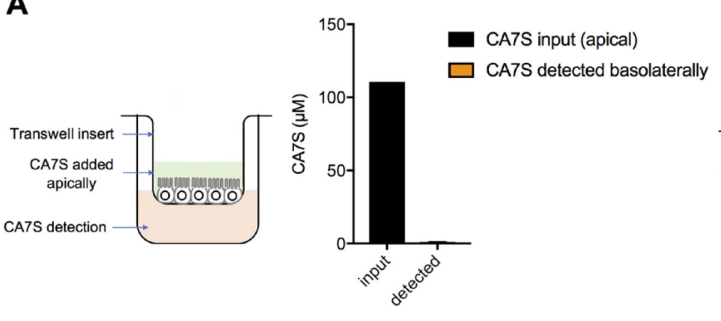

C
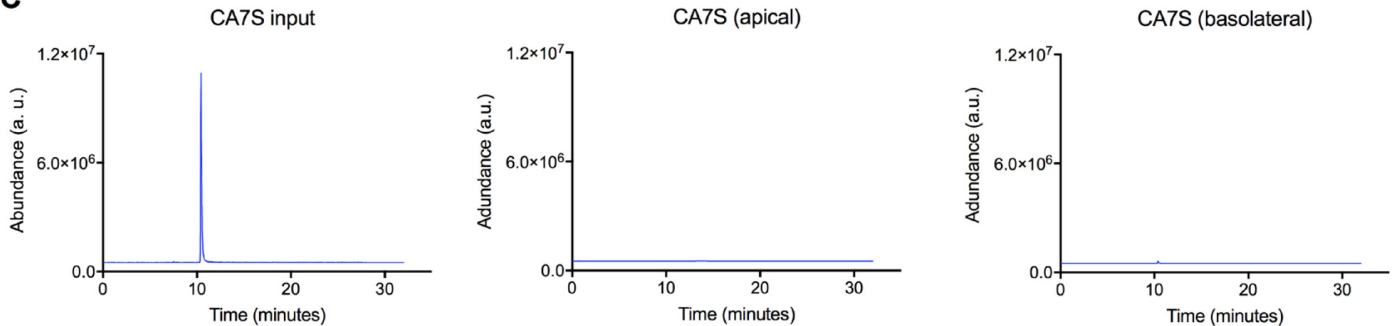

B

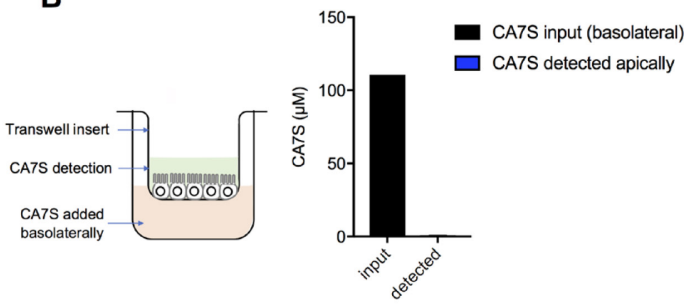

CA7S (basolateral)
D

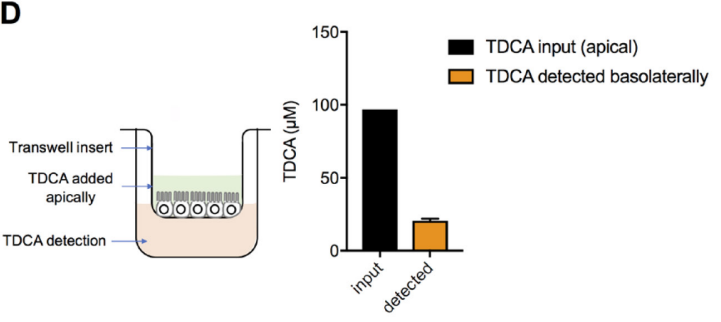

$\mathbf{F}$

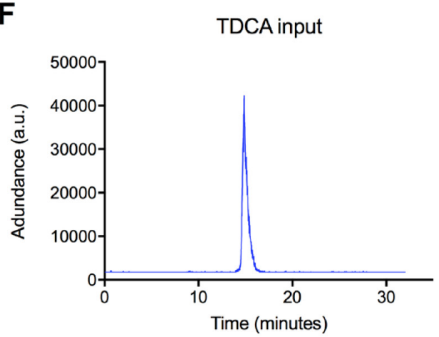

E

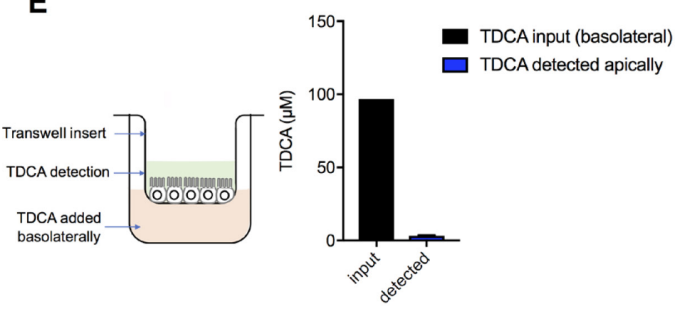

TDCA (apical)

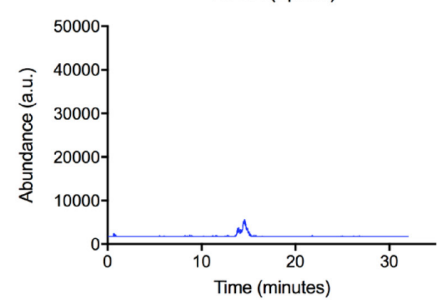

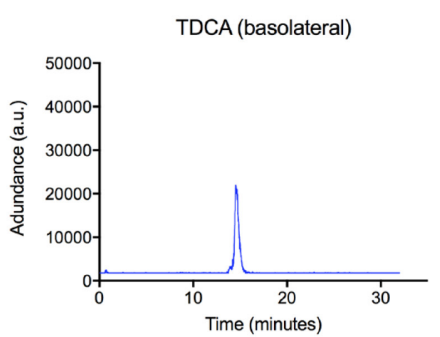

Figure 3. Testing of small molecule TGR5 agonists for gut-restricted properties using the co-culture transwell system. A. Cholic acid-7-sulfate (CA7S, $100 \mu \mathrm{M})$ is undetected in the basolateral chamber when treated apically in differentiated Caco-2 $+\mathrm{NCl}-\mathrm{H} 716$ cells. B. CA7S is undetected in the apical chamber when treated basolaterally $(100 \mu \mathrm{M})$ in differentiated Caco$2+\mathrm{NCl}-\mathrm{H} 716$ cells. These data (A and $\mathbf{B}$ ) suggest that CA7S does not pass through an intestinal epithelial monolayer and suggests that CA7S is gut-restricted ( $\geq 3$ biological replicates per condition). C. Representative UPLC-MS traces for A and B. D. Tauro-deoxycholic acid (TDCA), a naturally occurring TGR5 agonist, is detected in the basolateral chamber when treated apically $(100 \mu \mathrm{M})$ in differentiated Caco-2 + NCl-H716 cells. E. TDCA is detected in small amounts in the apical chamber when treated basolaterally $(100 \mu \mathrm{M})$ in differentiated Caco$2+\mathrm{NCl}-\mathrm{H} 716$ cells. These data ( $\mathbf{D}$ and $\mathbf{E}$ ) suggest that TDCA does pass through an intestinal epithelial monolayer and suggests that TDCA is not gut-restricted ( $\geq 3$ biological replicates per 
condition). F. Representative UPLC-MS traces for D and E. Quantification of CA7S and TDCA directly in media was performed by UPLC-MS-based detection as described previously (Yao et al., 2018; Chaudhari et al., 2021). All data are presented as mean \pm SEM.

8. To test whether molecules were toxic to the monolayer, cell viability can be measured using the MTT assay or CellTiter-Glo assay according to manufacturer's instructions and media provided in the respective kits (Figure 4A).

9. To test whether molecules damaged/affected intestinal barrier integrity, test transwells for epithelial integrity by Fluorescein isothiocyanate-dextran (FITC-D, 4 kDa) permeability assay.

10. Prepare $5 \mu \mathrm{M}$ FITC-D in PBS in the dark.

11. On Day 21 of the differentiation, remove media from the apical and basolateral compartments of the transwells.

12. Add $5 \mu \mathrm{M}$ FITC-D solution to the apical chamber of transwell inserts with cells, and add $500 \mu \mathrm{l}$ of PBS to the basolateral chamber.

13. Transfer plates to the tissue culture incubator, and incubate for exactly $20 \mathrm{~min}$.

14. After incubation, remove and discard transwell inserts.

15. Transfer $100 \mu \mathrm{l}$ of the basolateral chamber PBS into black 96-well plates.

16. Measure FITC-D by measuring fluorescence in a fluorescence plate reader with an excitation wavelength of $485 \mathrm{~nm}$ and an emission wavelength of $530 \mathrm{~nm}$ (Figure 4B).

A

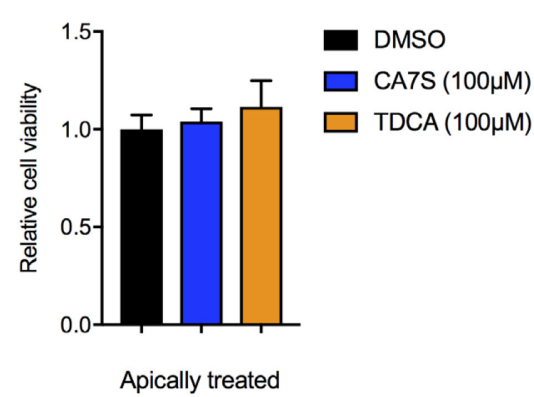

B

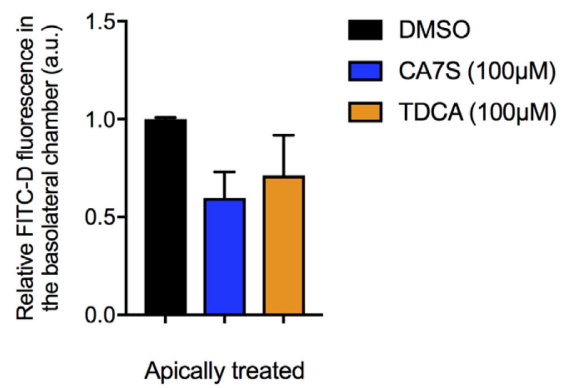

Figure 4. Testing of small molecule TGR5 agonists for toxicity and effect on intestinal permeability using the co-culture transwell system. A-B. Naturally occurring TGR5 agonists cholic acid-7-sulfate (CA7S) and tauro-deoxycholic acid (TDCA) do not affect cell viability (A) in the MTT assay, nor induce intestinal permeability (B) in the FITC-D assay in differentiated Caco$2+\mathrm{NCl}-\mathrm{H} 716$ cells. These results suggest that CA7S and TDCA are not toxic to human intestinal epithelial and enteroendocrine cells. When TDCA is treated apically, this compound does not cause epithelial toxicity or disrupt the gut barrier integrity, suggesting that accumulation of TDCA in the basolateral chamber (Figure 3D, F) may occur via active or passive transport. Data not marked with asterisk $\left(^{*}\right)$ is not significant. ( $\geq 3$ biological replicates per condition. A. CA7S vs. DMSO, $P=0.93$; TDCA vs. DMSO, $P=0.62$; B. CA7S vs. DMSO, $P=0.26$; TDCA vs. DMSO, $P=0.45$, one-way ANOVA followed by Dunnett's multiple comparisons test). MTT absorbance 
and FITC-D fluorescence readings performed as described previously (Chaudhari et al., 2021) at the ICCB-Longwood Screening facility at Harvard Medical School. All data are presented as mean \pm SEM.

\section{Data analysis}

Determine GLP-1 levels using the standard curve obtained from controls and reagents in the GLP1 EIA Kit according to manufacturer's instructions. Percentage GLP-1 secretion is calculated as follows:

Percentage GLP-1 secretion $=($ Total GLP-1 secreted into basolateral media $) /($ Total GLP-1 secreted into basolateral media) + Total GLP-1 in cell lysates) $\times 100$. Plot relative GLP-1 secretion as percentage $(\%)$ relative to the DMSO control.

\section{$\underline{\text { Notes }}$}

1. All cell culture experiments should be performed in a biosafety cabinet.

2. Pipette tips and serological pipettes used should be pre-sterilized and opened only in the biosafety cabinet.

3. Detection and quantification of bile acids is performed using UPLC-MS-based analysis as described previously (Yao et al., 2018; Chaudhari et al., 2021).

\section{Recipes}

1. Complete media

DMEM or RPMI media

$10 \%$ FBS

1× Pen Strep

2. Transfection media

$40 \mathrm{nM}$ siRNA or shRNA plasmid

OptiMEM media

Lipofectamine

3. Low-serum media for GLP-1 secretion

$500 \mathrm{ml}$ RPMI

$0.5 \%$ FBS

4. Cell lysis buffer (freshly prepared)

1\% TFA

$1 \mathrm{M} \mathrm{HCl}$

$5 \%$ formic acid

$1 \% \mathrm{NaCl}$ 


\section{Acknowledgments}

We thank members of the Devlin lab (Harvard Medical School-HMS) for helpful discussions. We would like to acknowledge the Clardy lab and the ICCB-Longwood Screening Facility at HMS for help with equipments and reagents. We thank the HMS Electron Microscopy core for help with SEM and TEM imaging. This work was supported by a an NIH MIRA grant (R35 GM128618) (A.S.D.), a Blavatnik Biomedical Accelerator at Harvard University grant (A.S.D.), a Quadrangle Fund for the Advancement and Seeding of Translational Research at HMS (Q-FASTR) grant (A.S.D.), an American Heart Association Postdoctoral Fellowship (S.N.C.), and a HMS Department of Biological Chemistry and Molecular Pharmacology Fellowship (S.N.C).

\section{Competing interests}

A.S.D. is a consultant for Takeda Pharmaceuticals and HP Hood.

\section{References}

1. Allaire, J. M., Crowley, S. M., Law, H. T., Chang, S. Y., Ko, H. J. and Vallance, B. A. (2018). The Intestinal Epithelium: Central Coordinator of Mucosal Immunity. Trends Immunol 39(9): 677-696.

2. Blutt, S. E., Crawford, S. E., Ramani, S., Zou, W. Y. and Estes, M. K. (2018). Engineered Human Gastrointestinal Cultures to Study the Microbiome and Infectious Diseases. Cell Mol Gastroenterol Hepatol 5(3): 241-251.

3. Cao, H., Chen, Z. X., Wang, K., Ning, M. M., Zou, Q. A., Feng, Y., Ye, Y. L., Leng, Y. and Shen, J. H. (2016). Intestinally-targeted TGR5 agonists equipped with quaternary ammonium have an improved hypoglycemic effect and reduced gallbladder filling effect. Sci Rep 6: 28676.

4. Chaudhari, S. N., Harris, D. A., Aliakbarian, H., Luo, J. N., Henke, M. T., Subramaniam, R., Vernon, A. H., Tavakkoli, A., Sheu, E. G. and Devlin, A. S. (2021). Bariatric surgery reveals a gut-restricted TGR5 agonist with anti-diabetic effects. Nat Chem Biol 17: 20-29.

5. Cristina, M. L., Lehy, T., Zeitoun, P. and Dufougeray, F. (1978). Fine structural classification and comparative distribution of endocrine cells in normal human large intestine. Gastroenterology 75(1): 20-28.

6. Duboc, H., Tache, Y. and Hofmann, A. F. (2014). The bile acid TGR5 membrane receptor: from basic research to clinical application. Dig Liver Dis 46(4): 302-312.

7. Eissele, R., Goke, R., Willemer, S., Harthus, H. P., Vermeer, H., Arnold, R. and Goke, B. (1992). Glucagon-like peptide- 1 cells in the gastrointestinal tract and pancreas of rat, pig and man. Eur J Clin Invest 22(4): 283-291. 
8. Gagnon, J. and Brubaker, P. L. (2015). NCl-H716 Cells. In: The Impact of Food Bioactives on Health: in vitro and ex vivo models. Verhoeckx, K., Cotter, P., Lopez-Exposito, I. et al. (Eds.). Cham (CH): 221-228.

9. Gonzalez, L. M., Williamson, I., Piedrahita, J. A., Blikslager, A. T. and Magness, S. T. (2013). Cell lineage identification and stem cell culture in a porcine model for the study of intestinal epithelial regeneration. PLoS One 8(6): e66465.

10. Harach, T., Pols, T. W., Nomura, M., Maida, A., Watanabe, M., Auwerx, J. and Schoonjans, K. (2012). TGR5 potentiates GLP-1 secretion in response to anionic exchange resins. Sci Rep 2: 430.

11. Hodge, R. J. and Nunez, D. J. (2016). Therapeutic potential of Takeda-G-protein-receptor-5 (TGR5) agonists. Hope or hype? Diabetes Obes Metab 18(5): 439-443.

12. Kim, K. S., Lee, I. S., Kim, K. H., Park, J., Kim, Y., Choi, J. H., Choi, J. S. and Jang, H. J. (2017). Activation of intestinal olfactory receptor stimulates glucagon-like peptide-1 secretion in enteroendocrine cells and attenuates hyperglycemia in type 2 diabetic mice. Sci Rep 7(1): 13978.

13. Lea, T. (2015). Caco-2 Cell Line. In: The Impact of Food Bioactives on Health: in vitro and ex vivo models. Verhoeckx, K., Cotter, P., Lopez-Exposito, I. et al. (Eds.). Cham (CH): 103-111.

14. Sato, H., Macchiarulo, A., Thomas, C., Gioiello, A., Une, M., Hofmann, A. F., Saladin, R., Schoonjans, K., Pellicciari, R. and Auwerx, J. (2008). Novel potent and selective bile acid derivatives as TGR5 agonists: biological screening, structure-activity relationships, and molecular modeling studies. J Med Chem 51(6): 1831-1841.

15. Yao, L., Seaton, S. C., Ndousse-Fetter, S., Adhikari, A. A., DiBenedetto, N., Mina, A. I., Banks, A. S., Bry, L. and Devlin, A. S. (2018). A selective gut bacterial bile salt hydrolase alters host metabolism. Elife 7: e37182. 